Théologiques

Théologiques

\title{
Bioéthique et inégalités sociales
}

\section{Márcio Fabri Dos Anjos}

Volume 7, numéro 1, printemps 1999

Les trente ans de la bioéthique

URI : https://id.erudit.org/iderudit/024971ar

DOI : https://doi.org/10.7202/024971ar

Aller au sommaire du numéro

\section{Éditeur(s)}

Faculté de théologie de l'Université de Montréal

\section{ISSN}

1188-7109 (imprimé)

1492-1413 (numérique)

Découvrir la revue

\section{Citer cet article}

Fabri Dos Anjos, M. (1999). Bioéthique et inégalités sociales. Théologiques, 7(1), 19-34. https://doi.org/10.7202/024971ar

\section{Résumé de l'article}

Par cet article nous voulons d'abord et avant tout poser la question du sens d'une réflexion en bioéthique dans le contexte des sociétés où les inégalités sociales sont poussées à leur extrême limite. Ancrée dans les prémisses de la théologie de la libération, notre réflexion veut identifier les sources du problème et tenter d'asseoir une méthodologie appropriée visant à intégrer dans la réflexion en bioéthique des considérations qui touchent aux inégalités sociales. d'utilisation que vous pouvez consulter en ligne.

https://apropos.erudit.org/fr/usagers/politique-dutilisation/ 
Théologiques 7/1 (1999) 19-34.

\title{
Bioéthique et inégalités sociales
}

\author{
Márcio FABRI DOS ANJOS* \\ Institut Alfonsianum d'éthique théologique \\ Sao Paulo, Brésil
}

Pour amorcer cette réflexion, je partirai d'une hypothèse empruntée à la science-fiction. Imaginons l'existence d'une vie intelligente en dehors de notre planète et pensons alors à ce que devrait être la réflexion bioéthique dans le cadre d'autres constellations. Un des principaux problèmes qui se poseraient viendrait des possibles différences tant dans les conditions de vie que dans les concepts utilisés pour en rendre compte. Pour résoudre les difficultés, on recourrait à une méthodologie qui chercherait à établir en quoi consiste la vie et comment elle se reproduit dans telles conditions. Ce matériel permettrait de commencer le travail de réflexion. Sans prétendre que les différences qui existent sur la planète Terre soient aussi mar quées que les diverses formes de vie que nous pourrions rencontrer dans d'autres galaxies, cette observation de départ nous invite à prendre conscience des différences existantes lorsque nous voulons aborder la question de la bioéthique.

Entre nous, sur terre, il ne s'agit pas tant de différences que d'inégalités. La Banque interaméricaine de développement (BID) est claire à ce propos. Une de ses récentes études conclut: "L'Amérique latine est le continent où l'on observe les plus grandes inégalités sociales. Et le pays où la pauvreté est la plus extrême est, selon les indicateurs, le Brésil. "Il est établi de façon précise que le dixième de la population la plus riche détient $47 \%$ du revenu natio-

* Ex-président de la Société brésilienne de théologie et de sciences de la religion, Márcio Fabri dos Anjos est directeur de l'Institut Alfonsianum d'éthique théologique de Sao Paulo. Son article a été traduit en français par Aldina da Silva et Hubert Doucet. 
nal, alors que le dixième de la population la plus pauvre se partage à peine $0,8 \%$ du revenu national (Natali, 1988). Dans ces conditions, que signifie penser la bioéthique en tenant compte d'inégalités si brutales?

Les réflexions que propose cet essai résultent du questionnement que suscitent les contradictions du contexte latino-américain au moment où la capacité de «produire" la vie n'a jamais été aussi grande. Elles se nourrissent du christianisme et trouvent dans la théologie de la libération ses sources d'inspiration. Le rapprochement que nous faisons entre les incidences du contexte social et la pensée bioéthique rend inévitable la discussion des questions méthodologiques. Une de nos préoccupations fondamentales consiste à faire de la bioéthique une "éthique de vie ", sans que nous nous perdions dans les distinctions théoriques de sa systématisation. Notre objectif ne consiste pas à réexaminer en détail une telle systématisation. Il cherche plutôt à asseoir la nécessité d'une méthodologie appropriée pour intégrer dans la réflexion bioéthique des considérations qui touchent aux inégalités sociales.

\section{Structures de la vie sociale et éthique de la vie}

En quoi les inégalités sociales peuvent-elles faire l'objet de la réflexion en bioéthique? Selon Warren $T$. Reich, les pionniers de la bioéthique (Potter et Hellegers) proposaient une bioéthique "globale " qui pourrait :

(1) relate to or involve the entire earth: a worldwide ethic for the good of the world; (2) entail the comprehensive inclusion of all ethical issues in the life sciences and health care (both the 'biomedical' and the 'environmental' issues of this classic debate); and (3) utilize a comprehensive vision of methods for approaching those issues : expansively incorporating all relevant values, concepts, modes of reasoning and disciplines. (Reich, $1995: 24)$

En conclusion de son article, Warren T. Reich donne sa position personnelle. Il considère qu'au plan universitaire : 
It would be preferable to make the following distinction in the title of this discipline: the use of the term 'bioethics' in its origi nal, global sense, as referring to the ethics of the life sciences and health care; and then use adjectives to specify particular areas of concern within bioethics. For example, one might speak of medical bioethics, clinical bioethics, or nursing bioethics without implying, with these terms, any particular approach. (Reich, $1995: 29-30$ )

Combiner la perception "globale» de la bioéthique avec les champs spécifiques qui émergent de cette globalité comporte cependant un risque. Il deviendrait alors facile de simplifier la compréhension des inégalités sociales et de les réduire à un champ particulier de préoccupation. Ainsi, on pourrait parler de bioéthique contextuelle latino-américaine.

Dans une vision qui part des pauvres, cette réduction pose problème. D'une part, les inégalités proviennent de champs différents qui cependant ne sont pas juxtaposés mais bien inter-reliés. D'autre part, une méthodologie scientifique appropriée sera en mesure de mettre à découvert l'articulation de l'inégalité. Sans doute, retrouvet-on plusieurs courants méthodologiques en bioéthique; la pluralité caractérise la bioéthique (Engelhardt, 1996:1). Si les pauvres sont au point de départ, l'inefficacité de ces multiples approches pour changer les inégalités est évidente et rend insatisfait. La critique fera aussi ressortir qu'une vision limitée à un seul champ, malgré son importance sous plusieurs autres aspects, peut devenir nuisible dans la mesure où cette vision est incapable de percevoir l'articulation plus large inter-reliant l'ensemble des champs.

Dans un texte polémique, Sandro Spinsanti applique cette critique à l'éthique médicale. Il écrit que celle-ci :

a un caractère idéologique (c'est-à-dire, masque et justifie les relations de pouvoir qui sont exercées concrètement dans la société et dans la profession); fut historiquement impuissante à prévenir de très graves abus, comme en témoigne le nationalsocialisme; et place le débat sur l'humanisation de la médecine à un plan superficiel, sans atteindre la racine des maux de la médecine. (Spinsanti, 1992 : 112-113) 
La critique de Spinsanti invite à un réexamen épistémologique des bases anthropologiques de la médecine et, en conséquence, de l'éthique qui la régit. Il en va généralement de même pour la bioéthique.

En effet, comme nous l'avons déjà mentionné, le faible niveau de vie des pauvres n'est pas, dans le contexte latino-américain, un phénomène passager. Il s'insère dans une trame sociale qui met au défi l'éthique de la vie. Même si le rappel insistant des statistiques qui montrent les données de la pauvreté peut déplaire à certains, il s'impose. Une enquête récente menée au Brésil démontre la chaîne de relations qui conduit à la reproduction des inégalités. En chiffres bruts, les données de 1998 du Programme des Nations Unies pour le développement (PNUD) établissent que les Brésiliens misérables, c'est-à-dire se situant en dessous du seuil de la pauvreté, composent $28,7 \%$ de la population adulte de près de 102 millions de personnes ayant plus de 16 ans (Datafolha, 1998:2). En utilisant une variété de critères, Datafolha classe environ $63 \%$ de la population dans la catégorie des exclus, leur revenu mensuel étant inférieur à $300,00 \$$ US. En additionnant tous les gains de la famille, les personnes classées misérables ont, en moyenne, un revenu mensuel d'à peine 215,00\$US (Toledo, 1998: 3).

Cette inégalité se reproduit, il importe de le remarquer, dans un contexte où sont exclues les possibilités d'éducation, de travail, d'habitation, d'alimentation, d'hygiène et autres besoins de base. Les données de Datafolha révèlent encore que $83 \%$ des pauvres classés miséreux possèdent une scolarité qui ne dépasse pas la quatrième année et sont considérés comme des « analphabètes fonctionnels». Cette dernière situation implique que ces personnes n'ont pas de travail, reproduisant ainsi le cycle de l'inégalité et de la misère. "Si, dans la pyramide sociale, le revenu est la ligne de partage des eaux, l'éducation, quant à elle, est la principale cause de la misère, ajoute l'enquête. »(Toledo, 1998: 3). Le manque d'éducation de base est sans doute une cause importante de misère, il n'en est cependant pas la principale. En effet, la viabilité des programmes d'éducation euxmêmes dépend de la spirale de l'inégalité qui se reproduit aussi par l'asymétrie de l'insertion des pays pauvres dans la politique économique internationale, les coûts des dettes extérieure et intérieure, la spéculation financière, la corruption et l'impunité. En même temps 
que le système économique nous pousse à toute vitesse vers la privatisation, les moyens de production, de plus en plus spécialisés, accroissent l'exclusion. Les pauvres n'ont d'autre choix que de se débrouiller eux-mêmes. Dans un anneau de cette chaîne se trouvent les enfants : selon les statistiques, le marché du travail brésilien employait en 1998 environ 3,8 millions d'enfants (Folha, 1998:2).

Ces statistiques démontrent que vie et santé résultent d'une construction sociale complexe. Se contenter de décrire la tâche de la bioéthique comme consistant à se préoccuper de la santé et du bien-être des individus ou à établir des lignes directrices pour faire face à des situations singulières est inacceptable. Ces statistiques obligent à reconnaître que toute une société se trouve gravement malade et reproduit une mauvaise qualité de vie. Du point de vue méthodologique, c'est le premier point à affirmer, les thèmes de la bioéthique doivent être analysés sous trois angles (Anjos, 1994: 141; $1996: 635)$. La dimension micro-sociale regroupe les questions et les thèmes issus des relations qui s'établissent entre des êtres qui sont proches. Ainsi en est-il des relations familiales, des relations du malade avec le médecin ou le personnel infirmier ou du lien qui s'établit entre la santé d'un individu et son environnement. Une dimension que nous qualifierons de mezzo-sociale concerne les préoccupations qui habitent les groupes de personnes, les questions qui naissent des réalités institutionnelles comme les hôpitaux, les groupes de recherche ou les relations qu'un groupe établit avec son environnement. La dimension macro-sociale est attentive aux grandes options socio-structurelles qui influencent directement ou indirectement la production de la vie et de la santé (Boff, 1993; Assman; Hinkelammert, 1989).

Une deuxième observation méthodologique est déjà consacrée par la théologie de la libération: face aux inégalités sociales, le questionnement doit s'élaborer à partir de la réalité sociale d'où émerge la réflexion. Le cas du Brésil permet à nouveau d'en vérifier la pertinence en ce qui concerne la bioéthique. Les inégalités placent côte à côte le sous-monde de la misère et l'oasis du bien-être avec toutes ses possibilités. Lorsque la bioéthique se fait à partir de la tranche des $10 \%$ des individus les plus riches du Brésil, il est inévitable que les problèmes et les questions abordés correspondent à ceux des riches. Les problèmes de la faim, du manque d'éducation, du 
chômage et de la pauvreté générale ne préoccupent pas une telle bioéthique. Il est certainement possible de regarder les problèmes bioéthiques qui se posent dans un hôpital sans remarquer les chemins tortueux qui empêchent les pauvres d'y avoir accès. Il est donc important de prendre conscience de cette «myopie » et de s'efforcer de donner à la méthodologie une dimension sociale pour ainsi écouter la voix des exclus.

Dans une telle démarche, la bioéthique ne revêt-elle pas un caractère idéologique? Changer l'« angle social " ne consiste-t-il pas à " changer d'idéologie "? La théologie peut contribuer à répondre à ces questions sans pour autant entrer dans le vieux débat à propos des idéologies. Il s'agirait d'approfondir le thème selon le point de vue des options et des motifs qui sous-tendent celles-ci. Est ainsi ouverte la question fondamentale sur les options qui, en dernière analyse, président à la rationalité bioéthique. Ces considérations nous conduisent à aborder un point de la plus haute importance.

\section{Quelle mystique pour quelle bioéthique?}

Peut-on parler de mystique en bioéthique? La racine étymologique grecque du mot (mys) permet de le comprendre ainsi : l'ensemble des forces et des motivations cachées et sous-jacentes unifiant les options et les critères de construction de sens et d'interprétation de la vie. La Bible décrit poétiquement la mystique du juste comme les racines d'un arbre planté prés des ruisseaux, qui produit des fruits dans la bonne saison et maintient ses feuilles vertes (Psaume 1,3). La racine latine renvoie à spiritualité, exprimant une dynamique sous jacente au soutien et à la promotion de l'action et de la réflexion.

En Amérique latine, aux dires de plusieurs théologiens, des constructions théoriques et pratiques qui ont l'apparence de réalités séculières, telles la macro-structure et les systèmes politiques inter nationaux, réfèrent à des fondements religieux (Assmann; Hinkelammert, 1989; Santa Ana, 1989; Assmann \& A., 1991; Sung, 1998). Cette interprétation n'est pas exclusive à la théologie; elle se vérifie aussi dans les lectures de la politique et de l'économie. Cette problématique se retrouve aussi en dehors des cadres de la théologie (Bénichou, 1948; Lovejoy, 1961; Hirschman, 1988). Autrement dit, la politique et l'économie ont aussi leurs mystiques ou spiritualités. 
Évidemment, ce sont des personnes concrètes qui mettent en œuvre des pratiques et pratiquent des théories. La condition humaine témoigne de notre carence anthropologique de "religion " (Bataille, 1993:21) et de spiritualité : nous sommes des êtres historiques obligés de chercher en dehors de nous, à commencer par la nourriture, le soutien nécessaire pour nous projeter dans l'être. A partir d'ici, soutiennent les théologiens de la libération, se pose, dans tous les secteurs de la vie, le défi de discerner le vrai Dieu de l'idole avec ses fausses promesses de vie, en d'autres mots, de différencier les dynamismes qui génèrent réellement la vie de ceux qui, d'une façon plus ou moins trompeuse, produisent la mort.

La bioéthique est un espace théorique où ces défis ne peuvent être ignorés. Inutile d'insister sur les manifestations de la mystique dans la bioéthique. Il suffit de regarder l'importance des motivations cachées qui nourrissent les différentes constructions de sens et des critères qui alimentent la réflexion. Il est intéressant de remarquer, par ailleurs, que l'International Association of Bioethics maintient dans ses congrès internationaux une section intitulée "Bioethical Discourse \& Religions ».

C'est dans ce contexte que nous cherchons à préciser la contri bution de la théologie de la libération. Il s'agit de voir comment la mystique peut nourrir la bioéthique. La théologie chrétienne a parti cipé à la naissance de la bioéthique, particulièrement par ses contri butions et réflexions autour de l'éthique médicale. Dans les discussions actuelles sur la bioéthique, sa contribution apparaît, au moins, à deux niveaux principaux. La théologie se propose d'abord d'élargir le sens de la vie: dépassant l'acte de naissance, elle se préoccupe aussi des diverses relations humaines, du plaisir, de la souffrance, de la mort. En plus, elle met de l'avant des critères et des propositions éthiques correspondant à cette vision de la vie pour en guider la conduite. Dans la construction de la bioéthique, le sens de la vie et les critères éthiques sont deux coordonnées indispensables.

Il peut être utile de rappeler quelques éléments à la base d'une spiritualité de libération (Boff, 1987; Casaldáliga \& Vigil, 1993) pouvant servir la bioéthique. Dans ses grandes lignes, la théologie de la libération voit la présence de Dieu dans le monde comme le Créateur (Père-Mère) qui fait de l'être humain un participant au 
processus de création tout comme à la conduite du monde et de luimême vers une plénitude de vie. Les potentialités humaines sont des dons appelés à croître. Mais, parce qu'elle est toujours en apprentissage, l'humanité entière, engagée dans la trame des relations et dans l'avancement des sciences et des technologies, utilise ses potentialités avec beaucoup d'ambiguité. Dieu montre que le chemin de la vie passe par des relations de solidarité, de justice et d'amour alors que celui de l'égoïsme, de la prépotence et du désengagement offre de fausses promesses et des images (idoles) de plénitude de vie qui, en réalité, engendrent la Mort, la servitude et l'exclusion.

La vie est ainsi comprise comme un grand processus de libération. Nous ne sommes pas encore libres; nous sommes des apprentis de la liberté. Et cet apprentissage se réalise à travers l'avancement de la science et la sagesse de la vie. Celle-ci se solidifie dans le mouve. ment d'amour solidaire qui peut parvenir à vaincre les attitudes d'égoïsme et de désengagement. Dans les relations sociales, cet amour solidaire correspond au développement de la justice sociale capable de surmonter les dominations, les exclusions et la destruction de la vie.

La méthode théologique qui répond à cette vision met en évidence trois moments interactifs du processus de libération: le moment herméneutique où s'établissent les critères et les références fondamentales; le moment de l'analyse de la réalité où s'analyse la vie à la lumière des critères retenus; le moment des pratiques de libération où se vit la mystique des critères et de ses exigences. Théorie et pratique sont interactives. Cette méthode favorise une interprétation de la Bible qui est particulièrement adéquate pour saisir la présence du salut amoureux de Dieu dans la dialectique de la vie socio-culturelle, politique et économique. Elle montre comment les ambiguïtés envahissent non seulement le cœur des individus, mais aussi les structures sociales. Elle inclut une relecture des dimensions sociopolitiques de la mort de Jésus dans son geste suprême d'amour solidaire. En même temps, la méthode facilite le rapprochement avec les sciences pour réaliser des analyses capables de mettre à jour les multiples défis et projets de libération: surmonter les naïvetés, découvrir les structures de domination et d'exclusion, rendre l'amour efficace. 
La recherche d'une relation étroite et interactive entre foi et réalité, théorie et pratique, idéal et réel caractérise cette méthode théologique. Tout en faisant de la mystique de l'amour son principe fondamental, cette méthode procède de façon intelligente et effi cace. La pauvreté, les asymétries sociales et l'exclusion expriment, surtout depuis les années 1970, le grand défi qui confronte l'huma. nité en route vers la libération. L'option préférentielle pour les pauvres signifie que la libération est en devenir et qu'elle forme des communautés. À mesure que s'opère la libération, se précisent les différents visages de l'appauvrissement qui passent par les relations économiques, politiques, ethniques et culturelles. Les dimensions écologiques et environnementales y trouvent aussi place (Boff : 1993). Si l'expression «théologie de la libération " n'est plus aussi utilisée, les traces caractéristiques de sa méthodologie demeurent visibles.

Voici, brièvement présentés, les principaux éléments d'ordre mystique qu'apporte cette approche libératrice à la bioéthique :

a) une cosmovision de la vie : recevoir comme un don la vie humaine qui doit être vécue en société, dans l'histoire humaine et au-delà du temps;

b) un contexte au fondement des relations: les relations entre les vivants signifient davantage qu'une existence commune pacifique. Les humains doivent se comporter comme des partenaires de vie, en appelant à la solidarité pour réduire les asymétries;

c) un horizon de sagesse : le secret de la relation humaine réside dans l'expérience de la gratuité et du partage de vie. Un proverbe populaire résume cette mystique ainsi : "Peu, avec Dieu, est beaucoup; beaucoup, sans Dieu, est rien. " L'option préférentielle pour les pauures exprime cette sagesse : la vie se réalise sur un fond d'horizon qui surmonte la mort;

d) une méthode : rendre efficaces dans l'histoire, l'amour et la solidarité; analyser la réalité intégrale des personnes, leurs contextes et leurs dimensions (richesses, carences et prisons) en les considérant comme un défi sur la voie de la libération mais sans naïvetés; dialoguer avec les sciences sans oublier que celles-ci ne sont pas neutres. 
e) un programme d'action : mettre de l'avant les dimensions communautaires de la vie pour renforcer l'appel humanitaire et solidaire à agir humainement; accroître, de façon théorique et pratique, les efforts de la communauté solidaire en faveur de la vie et des défis qu'elle doit affronter.

Dans une mystique de libération, la bioéthique se fait profondément solidaire du cri de douleur de l'humanité et de la planète. Elle se rend disponible pour analyser à fond leurs inquiétudes et leurs souffrances. Elle offre un espace de rencontre et soutient les nombreux efforts qui sont requis pour aider les personnes prêtes à redonner espoir à tout être vivant, surtout aux appauvris et exclus.

\section{Réévaluation des principes}

Pour manifester l'intérêt que représentent ces diverses dimensions de libération, nous voudrions les mettre en relation directe avec ce que l'on appelle les principes de la bioéthique. Nous ne reprendrons ici ni une brève exposition ni une appréciation critique des principes de la bioéthique (Beauchamp et Childress, 1994; DuBose, Hamel et O'Connel, 1994). Les concepts connus d'autonomie, de bienfaisance, de non-malfaisance et de justice nous permettront d'expliciter quelques aspects libérateurs qui sont importants dans le contexte latino-américain marqué par les inégalités.

Cette brève relecture des principes de la bioéthique à la lumière d'une méthode de libération mettra en relief comment ils peuvent éclairer la face sociale de la réalité humaine. Elle accordera une attention particulière aux dimensions socio-culturelles, cherchant ainsi à dépasser une interprétation des principes limitée aux questions médico-hospitalières. L'" option pour les pauvres" est ici implicite puisque la méthode utilisée fait apparaître le visage des personnes les plus démunies.

1. Le principe d'autonomie est surtout porteur de dimensions philosophiques. Il ne peut cependant faire abstraction des réalités sociologiques qui soutiennent les autonomies, les menacent ou les annulent. Il n'établit pas seulement les bases d'une éthique du devoir mais souligne la dignité de la personne humaine. C'est pourquoi il ne peut se conjuguer seulement en termes d'individus mais aussi en 
termes de groupes et de communautés. La bioéthique ne peut ignorer l'identité des divers sujets sociaux et les inégalités qui les assujettissent. L'une des principales conclusions se dégageant habituellement de l'analyse du principe concerne la nécessité du «consentement informé ». Il s'agit d'un grand pas en avant quand il devient «consentement libre et éclairé » (Romero et Fortes, 1998: 53). Une étude récente faite sur l'expérience actuelle de l'Amérique latine nous amène à tirer une conclusion différente (Anjos, 1998). En effet, l'analyse sociologique montre que l'autonomie des sujets s'inscrit dans un réseau social complexe qui ne permet pas de comprendre ou de postuler l'autonomie comme se limitant aux seules relations entre le chercheur et le sujet de recherche ou entre le médecin et son patient. L'autonomie des personnes passe aujourd'hui par le savoir technologique relié au pouvoir politique et économique. Qui ne possède pas le pouvoir est vulnérable et qui est vulnérable s'expose à voir son autonomie bafouée par des manques de respect grossiers et rusés.

La vulnérabilité ne touche pas que des individus et des groupes, mais aussi des groupes ethniques, des populations entières et même toute une nation. Dans chacune de ces catégories, les exemples ne manquent pas. Penser l'autonomie exige de ne pas la séparer du concept clé que représente la vulnérabilité. Il est vrai qu'en bioéthique l'autonomie est un principe théorique et non un champ d'analyse sociologique. Comme diraient certains; moment d'éthique théorique et non d'éthique appliquée. Pourtant, quand les champs de son application ne sont pas bien définis, le principe devient facilement un piège : les individus dépourvus ne sont pas protégés et la pensée libérale qui se refuse à considérer les défis que pose la réalité sociale est favorisée. $\mathrm{Vu}$ sous l'angle de la libération, le principe d'autonomie ne se réduit pas au seul respect du consentement libre et éclairé. Il offre un défi à la bioéthique, celui de l'autonomie à construire et à renforcer là où celle-ci n'existe pas. Et là où l'autonomie n'existe pas, on ne peut se contenter de voir la vulnérabilité comme un respect passif, elle doit devenir un défi à construire la solidarité.

2. Les principes de bienfaisance et de non-malfaisance sont souvent considérés ensemble, malgré l'insistance que mettent Beauchamp et Childress à les distinguer (1994). Leur application couvre un vaste champ dans le domaine de la biomédecine. Selon une très bonne étude, publiée récemment à propos du contexte latino-américain, 
« le principe de bienfaisance sera, pour une société en voie de développement, celui qui devra orienter les activités et les décisions du personnel de santé comme citoyens conscients de leur rôle et de leur responsabilité personnelle et sociale " (Kipper et Clolet, 1998: 50).

Dans une perspective de libération, ces principes prennent une configuration structurelle, sans évidemment que soit rejetée leur application dans les domaines biomédicaux et interpersonnels. L'étude de Kipper et Clolet (1998: 40-41) parle même de la proximité entre les principes cités et une éthique appliquée à l'environnement selon les études de Hans Jonas (1995) et de Peter Singer (1991). Cependant, dans le cadre de la bioéthique, il est plutôt intéressant de rapprocher ces deux principes des décisions politiques qui touchent plus directement la santé publique. À ce sujet, le Brésil fait une expérience positive, celle du Conseil national de la santé où se retrouvent des représentants de nombreux groupes qui discutent, sur une base régulière, des décisions politiques qui concernent l'avenir de la santé dans la société brésilienne tant du point de vue de sa totalité que de ses différentes composantes. Dans un pays comme le Brésil, les différences sociales criantes génèrent des attentes diverses quant à la longévité et à la qualité de la vie. Les relations de bienfaisance et de malfaisance passent ainsi par une construction sociale plus large que la bioéthique ne peut ignorer.

3. Depuis l'étude classique de John Rawls (1971), les travaux sur le principe de justice sont innombrables et précieux (voir, par exemple, Veatch, 1986; Berlinguer, 1993; Siqueira, 1998). Les différents courants d'interprétation témoignent non seulement de la diversité des idéologies, mais aussi, comme le note Engelhardt, des «frustrations devant la finitude" (Engelhardt, 1996: ch. 8). Les considérations théologiques seront ici brèves.

Dans la perspective de la libération, la justice est un concept fondamental. Dans sa dimension théologique, issue de la tradition judéo-chrétienne, le concept s'enracine dans la condition existentielle de tous les êtres humains qui sont considérés comme des semblables. Dieu est Père-Mère de tous et tous sont frères et sœurs : telle est la justice selon Dieu. L'habitat propice à la vie est l'amour solidaire. Pour la théologie chrétienne de la libération, la justice demande que la bioéthique dépasse une conception simplement distributive qui se limite aux relations de coûts-bénéfices et à l'alloca- 
tion des ressources. Une conception légitime de la justice distributive en est une d'équité guidée par l'amour solidaire: les nécessités et les carences éprouvées par les êtres humains deviennent le critère de priorité. L'option préférentielle pour les pauvres, défendue par la théologie de la libération, appartient à cet horizon de justice.

Cette approche est inséparable d'une mystique ou d'une spiritualité qui ne peut se transformer en norme ou en loi. Elle ouvre une voie que toute l'humanité est invitée à prendre et à apprendre.

\section{Conclusion}

Les inégalités sociales posent à la bioéthique d'intéressantes questions méthodologiques. Plutôt que d'entrer dans l'analyse systématique de ces questions, nous avons préféré, dans une perspective de théologie de la libération, proposer des éléments utiles à la construc tion d'une éthique de vie face aux inégalités. Deux éléments particulièrement importants émergent de la réflexion que nous avons proposée. Le premier donne à la bioéthique une capacité d'analyse structurelle qui provient de la mise à nu de la logique de reproduction de l'inégalité sociale. La bioéthique a tendance à traiter isolément les différents champs d'application qui la composent, comprenant mal les diverses interrelations qui existent entre ces champs et surtout demeurant insensible aux facteurs qui sont responsables des inégalités. C'est ainsi que la complaisance devant les structures sociales injustes qui produisent la mauvaise qualité de la vie et même la mort guette la bioéthique. Sans cette capacité d'analyse structurelle, la critique se montre inefficace pour comprendre les grandes contradictions de la vie d'aujourd'hui.

D'un autre côté, qui s'intéresse aux pauvres, aux plus faibles, aux nécessiteux? La suppression des inégalités sociales pourrait peut-être se réaliser par la suppression de l'un des deux pôles de l'inégalité. Les propositions, plus ou moins claires, ne manquent pas. Une des contributions les plus fortes de la théologie de la libération tient dans le développement d'une mystique qui perçoit le prochain amoureuse ment. Dans ce contexte, les inégalités se transforment en clameur. Sans amour, la bioéthique réduit au silence sa pouvoir d'indignation devant les contrastes qui nous apparaissent sans solution. Avec l'amour, elle est poussée à réaliser une transformation sociale efficace. La bioéthique se fait alors avec la raison et le cœur. 


\section{Bibliographie}

ANJOS, M. Fabri, Bioethics in a liberationist key, dans Edwin R. DUBOSE, Ron HAMEL, Laurence J. O'CONNELL, A Matter of principles? Ferment in U.S. Bioethics. Valleyf Forge, Trinity Press Internaitonal, 1994, pp. 130-147.

ANJOS, M. Fabri, «Le pouvoir, l'éthique et les pauvres dans la recherche en génétique humaine ", Concilium 275 (1998) 99-110.

ANJOS, M. Fabri, "Medical ethics in the developing world: a Liberation Theology perspective", Journal of Medicine and Philosophy 21 (1996) 629-637.

ASSMANN, Hugo (ed.), René Girard com teólogos da libertaçãio. Un diálogo sobre idolos e sacrifícios. Petrópolis, Ed. Vozes ,1991.

ASSMANN, Hugo; HINKELAMMERT, Franz. A Idolatria do Mercado. Ensaio sobre Economia e Teologia. Petrópolis, Ed. Vozes, 1989.

BATAILlE, George, Teoria da Religião. S. Paulo, Ed. Ática, 1993 (Théorie de la religion. Paris, Gallimard, 1973).

BEAUCHAMP, T.L. \& CHILDRESS, J.F., Principles of Biomedical Ethics. New York, Oxford University Press, 1994, $4^{\mathrm{a}}$.

BÉNICHOU, Paul. Morales du grand siècle. Paris, Gallimard, 1948.

BERLINGUER, G., Questões de vida. S. Paulo, HICITEC-CEBES, 1993.

BOFF, Leonardo, Vida segundo o Espírito. Petrópolis, Ed. Vozes, 1987, $4^{\mathrm{a}}$.

BOFF, Leonardo, Ecologia, mundializoção, espiritualidade. A emergência de um novo paradigma. S. Paulo, Ed. Atica, 1993.

CASALDÁlIGA, Pedro; VIGIL, José Maria. Espiritualidade de Libertação. Petrópolis, Ed. Vozes, 1993. 
DATAFOLHA, Mapa da Exclusão dans Folha de S. Paulo 30-XI1998, especial A 1, 1-8.

ENGELHARDT, H. Tristram, The Fondations of Bioethics. New York, Oxford University Press, 996.

FOLHA DE S. PAULO 30/11/1998 : "Renda Minima " (editorial) caderno 1, pg. 2.

HIRSCHMAN, Albert O., As Paixões e os Interesses. Argumentos políticos a favor do Capitalismo antes de seu triunfo. Rio de Janeiro, Ed. Paz e Terra, 1988.

JONAS, Hans, El principio de responsabilidad: ensayo de una ética para la civilización tecnológica. Barcelona, Ed. Herder, 1995.

KIPPER, Délio; CLOTET Joaquim. Princíos da Beneficência e Nãomaleficêncial dans Ibiapina, Sérgio; Garrafa, Volney; Oselka, Gabriel. Iniciação à Bioética. Publicação de Conselho Federal de Medicina, Brasília, 1998, pp. 37-51.

LOVEJOY, Arthur O., Reflections on Human Nature. Baltimore, The Johns Hopkins Press, 1961.

MUÑOZ, Daniel R.; FORTES, Paulo A.C., O princípio da autonomia $e$ do consentimento livre e esclarecido dans IBIAPINA, Sérgio; GARRAFA, Volney; OSELKA, Gabriel. Iniciação à Bioética. Publicação do Conselho Federal de Medicina, Brasília, 1998, pp. 53-70.

NATALI, J. Batista. Brasil é o país mais desigual da AL dans Folha de São Paulo 14-XI- 1998, 1,12.

RAWLS, John. A theory of Justice. Cambridge, MA, Harvard University Press, 1971.

REICH, Warren Th., "The word 'bioethics': the struggle over its earliest its meanings », Kennedy Institute of Ethics Journal 5/1 (1995)19-34. 
SANTA ANA, Júlio. O Amor e as Paixões. Crítica Teológica à Economia Política. Aparecida, Ed. Santário, 1989.

SINGER, Peter, A companion to ethics. Cambridge, MA, Blackwell, 1991.

SIQUEIRA, Eduardo. O princípio da Justiça dans IBIAPINA, Sérgio; GARRAFA, Volney; OSELKA, Gabriel. Iniciação à Bioética. Publicação do Conselho Federal de Medicina, Brasília, 1998, pp. 71-80.

SPINSANTI, Sandro, Curar o Homem Todo. S. Paulo, Paulinas, 1992.

SUNG, Jung Mo. Idolatria: uma chave de leitura da economia contemporânea? In BRITO, Enio J.C. \& GORGULHO, Gilberto (ed.). Religião ano 2000. S. Paulo, Ed. Loyola, 1998.

TOLEDO, J. Roberto. Mapa da exclusão dans Folha de S. Paulo 30-XI1998, especial A 1, 3.

VEACHT, Robert. The foundations of justice. New York, Oxford University Press, 1986. 